\title{
Genetic analysis of Helicobacter pylori clinical isolates suggests resistance to metronidazole can occur without the loss of functional $r d x A$
}

\author{
So Yeong Kim ${ }^{1,5}$, Young Min Joo ${ }^{1,5}$, Hak Sung Lee ${ }^{2}$, In-Sik Chung ${ }^{2}$, Yun-Jung Yoo ${ }^{1}$, D Scott Merrell ${ }^{3}$ \\ and Jeong-Heon Cha ${ }^{1,4}$
}

Resistance to metronidazole (MTZ) in Helicobacter pylori is associated with mutations in $r d x A$, encoding an oxygen-insensitive NADPH nitroreductase, and mutations in frxA, encoding a NAD(P)H-flavin oxidoreductase. Despite this association, the strict correlation of MTZ resistance with mutations in $r d x A$ or frxA is still controversial. In this study, $r d x A$ allelic replacement was used to distinguish resistance-associated nucleotide mutations from the natural genetic diversity of $H$. pylori. Replacement with truncated $r d x A$ resulted in MTZ resistance, whereas replacement with missense-mutated $r d x A$ from resistant clinical isolates failed to yield MTZ resistance. Thus, although truncation of $r d x A$ confers MTZ resistance in G27 H. pylori, MTZ resistance found in other clinical isolates is not due to the identified amino-acid substitutions. Three of our MTZ-resistant clinical isolates expressed functional $r d x A$ and two of these also encoded full-length frxA. Therefore, MTZ resistance can arise in $H$. pylori possessing functional $r d x A$, suggesting that other factors are involved in MTZ resistance.

The Journal of Antibiotics (2009) 62, 43-50; doi:10.1038/ja.2008.6; published online 9 January 2009

Keywords: antibiotic resistance; FrxA; genetics; Helicobacter pylori; metronidazole; RdxA

\section{INTRODUCTION}

Since Helicobacter pylori was discovered in human gastric biopsy specimens in $1982,{ }^{1}$ it has been recognized as a significant contributing factor to the development of a number of gastric disorders. These include gastritis, ulcer disease and two distinct forms of gastric cancer, gastric adenocarcinoma and B-cell-mucosa-associated lymphoma. ${ }^{2-6}$ H. pylori chronically infects more than $50 \%$ of the world's population, making it one of the most common bacterial infections worldwide. Given the large number of infected individuals and the strong association of $H$. pylori infection with gastric disease, $H$. pylori exerts a tremendous medical burden. This fact makes effective treatment regimens extremely important.

Metronidazole (MTZ), a synthetic nitroimidazole, was a critical ingredient of the first successful $H$. pylori therapy and remains a major component of several multidrug therapies that contain a proton pump inhibitor and a combination of two or more antibiotics (MTZ, clarithromycin, amoxicillin or tetracycline). ${ }^{7,8}$ A major obstruction to successful $H$. pylori treatment is the presence of antibiotic-resistant strains. In fact, resistance to MTZ is common among clinically isolated H. pylori, with frequencies ranging from $10 \%$ to more than $90 \%$, depending on the geographic region and patient group. ${ }^{9,10}$ MTZ resistance is of clinical significance because it decreases the effectiveness of popular and affordable MTZ-containing anti-H. pylori therapies. ${ }^{11,12}$ In addition, as MTZ is also used against a wide variety of prokaryotic and eukaryotic pathogens, ${ }^{13-15}$ understanding the whole range of MTZ resistance mechanisms utilized by $H$. pylori may shed light on similar pathways in other clinically significant microbes.

The mechanism of antimicrobial action of MTZ has been investigated in anaerobic microbes. ${ }^{13,16,17}$ The cytotoxicity of MTZ is due to unstable intermediates that damage the DNA, resulting in strand breakage, helix destabilization, unwinding and ultimately cell death. ${ }^{18,19}$ Moreover, the anti-microbial action of MTZ is dependent on its reductive activation by the redox system of the target cell. ${ }^{20}$

This study focuses on the mechanisms of susceptibility and resistance of $H$. pylori to MTZ. In this bacterium, MTZ resistance has been shown to be strongly associated with mutations in $r d x A$,

${ }^{1}$ Department of Oral Biology, Oral Science Research Center, BK21 Project, Yonsei University College of Dentistry, Seoul, Republic of Korea; ${ }^{2}$ Division of Gastroenterology, Department of Internal Medicine, College of Medicine, The Catholic University of Korea, Seoul, Republic of Korea; ${ }^{3}$ Department of Microbiology and Immunology, Uniformed Services University of the Health Sciences, Bethesda, MD, USA and ${ }^{4}$ Department of Applied Life Science, The Graduate School, Yonsei University, Seoul, Republic of Korea Correspondence: Dr DS Merrell, Department of Microbiology and Immunology, Uniformed Services University of the Health Sciences, 4301 Jones Bridge Road, Bethesda, MD 20814, USA.

E-mail: dmerrell@usuhs.mil or Dr J-H Cha, Department of Oral Biology, Oral Science Research Center, BK21 Project, Yonsei University College of Dentistry, Seoul, Republic of Korea.

E-mail: jcha@yuhs.ac

${ }^{5}$ These authors contributed equally to this work.

Received 22 July 2008; accepted 10 October 2008; published online 9 January 2009 
a chromosomal gene encoding an oxygen-insensitive NADPH nitroreductase, and mutations in $f r x A$, a chromosomal gene encoding a $\mathrm{NAD}(\mathrm{P}) \mathrm{H}$-flavin oxidoreductase. However, the absolute association of H. pylori MTZ resistance with mutations of $r d x A$ or frxA is still a debated topic. $^{21-23}$ To conclusively prove this association, the contribution of specific $r d x A$ or frxA mutations to MTZ resistance needs to be confirmed using classical genetic techniques. However, genetic tools and strategies for manipulation of $H$. pylori still lag behind those available for other model organisms. In this study, both $r d x A$ and $f r x A$ genes from clinically isolated $H$. pylori strains were analyzed for sequence variation. To determine whether specific identified mutations in $r d x A$ were responsible for the resistance patterns associated with the clinical isolates, allelic exchange was used to replace the $r d x A$ gene of an MTZ-sensitive wild-type $H$. pylori strain with the $r d x A$ gene from most of the clinical isolates. Minimum inhibitory concentrations (MICs) of MTZ of these $r d x A$-replaced transformants were then determined to evaluate the contribution of the $r d x A$ mutations to MTZ resistance.

\section{MATERIALS AND METHODS}

\section{$H$. pylori strains and culture condition}

A total of 10 clinical $H$. pylori isolates, five MTZ-sensitive (S1-S5) and five MTZ-resistant (R1-R5), were obtained from patients at the Division of Gastroenterology, Department of Internal Medicine, College of Medicine, The Catholic University of Korea, Seoul, Republic of Korea. A written informed consent was received from each patient. The Institutional Review Board of Human Research at the Catholic University of Korea approved the protocol. The 3 previously characterized strains, ATCC43504, 26695 and G27, ${ }^{24}$ and the $10 \mathrm{H}$. pylori clinical isolates were cultured on Columbia blood agar plates (Difco, Detroit, MI, USA) containing $5 \%$ horse blood (Oxoid, Basingstoke, UK) in $10 \% \mathrm{CO}_{2}$ at $37^{\circ} \mathrm{C}$.

\section{Determination of MIC}

MIC was defined as the lowest concentration of MTZ that completely inhibited the growth of the inoculum. MICs were determined by the $E$-test method (AB Biodisk, Solna, Sweden) and agar dilution method. ${ }^{25}$ The $E$-test was performed on Columbia blood agar plates containing 5\% horse blood according to the manufacturer's instructions. The agar dilution method was performed on Columbia blood agar plates containing $5 \%$ horse blood as described earlier. ${ }^{25}$ Frozen bacterial stocks were streaked on Columbia blood agar and incubated for 3 days. Cells from a few colonies from these initial plates were then restreaked on fresh Columbia blood agar plates and incubated for one more day. The resulting exponentially growing cells were suspended in phosphatebuffered saline; series of 10 -fold dilutions of these cell suspensions were prepared, and $10 \mu \mathrm{l}$ of each dilution was spotted on freshly prepared Columbia blood agar containing various concentrations $(0,0.2,0.5,1.5,3,5,8,16,32,64$ or $256 \mu \mathrm{g} \mathrm{ml}^{-1}$ ) of MTZ (Sigma Chemical Co., St Louis, MO, USA). The plates were incubated for 3 days. MTZ-resistant H. pylori ATCC43504 and MTZsensitive 26695 were used as control strains. MTZ resistance was considered to be attained at an MIC of $>8 \mu \mathrm{g} \mathrm{ml}^{-1}$. ${ }^{21}$

\section{Cloning of $r d x A$ and $f r x A$ genes of clinical $H$. pylori isolates}

Chromosomal bacterial DNA was extracted using the Wizard Genomic DNA purification kit (Promega, Madison, WI, USA). PCR amplifications of $r d x A$ and frxA were carried out in a DNA thermal cycler (Biometra, Goettingen, Germany) using the Expand High Fidelity PCR System (Roche Applied Science, Mannheim, Germany) as follows: 1 cycle at $95^{\circ} \mathrm{C}$ for $2 \mathrm{~min} ; 30$ cycles of $95^{\circ} \mathrm{C}$ for $30 \mathrm{~s}, 55^{\circ} \mathrm{C}$ for $30 \mathrm{~s}$ and $72{ }^{\circ} \mathrm{C}$ for $30 \mathrm{~s}$ and a final elongation step at $72{ }^{\circ} \mathrm{C}$ for $10 \mathrm{~min}$. The oligonucleotide PCR primers used to amplify an 851-bp fragment containing the entire $r d x A$ open reading frame were a forward primer $\left(5^{\prime}-\right.$ aatttgagcatggggcaga- $\left.3^{\prime}\right)$ and a reverse primer ( $5^{\prime}$-gaaacgcttgaaaacacccct- $\left.3^{\prime}\right)$. Similarly, a forward primer ( $5^{\prime}$-ccatcgatatggacagagaacaagtgg- $\left.3^{\prime}\right)$ and a reverse primer ( $5^{\prime}$-gctaacagcgttttattcaatc- $3^{\prime}$ ) were used to amplify the 675-bp fragment containing the entire frxA open reading frame. The amplified PCR fragments were extracted from 1\% agarose gels using the QiaQuick Gel Extraction kit
(Qiagen, Hilden, Germany), ligated into the pGEM-T-easy vector (Promega) and transformed into Escherichia coli DH5 $\alpha$, generating plasmid pRDXA-G27, $-43504,-S 1$ to $-S 5$ and -R1 to -R5, and pFRXA-G27, -43504, -S1 to -S5 and -R1 to $-\mathrm{R} 5$.

Nucleotide sequence analysis of $r d x A$ and $f r x A$ of G27, ATCC43504 and clinically isolated $H$. pylori

DNA sequences of $r d x A$ and frxA of G27, ATCC43504 and the clinical isolates were determined for both strands of the inserts of pRDXA and pFRXA series (Cosmogenetech, Seoul, Republic of Korea) using T7 and SP6 primers. The resulting DNA sequences were analyzed by the vector NTI v9.1 (Invitrogen, Carlsbad, CA, USA) and Sequencher v4.5 (Gene Code, Ann Arbor, MI, USA) programs. The GenBank accession numbers for the sequences reported in this paper are EF444879-EF444880, EF471983-EF471992 and EF521388-EF521397.

\section{Generation of $r d x A$ constructs for natural transformation}

A 1.4-kb kanamycin $(\mathrm{Km})$ resistance cassette $(a p h A-3)$ was PCR amplified from pILL600 ${ }^{26}$ using a forward primer ( $5^{\prime}$-ccaagcttggcgtatcacgaggccctttcg- $\left.3^{\prime}\right)$ and a reverse primer ( $5^{\prime}$-ccaagcttctaaaacaattcatccag- $\left.3^{\prime}\right)$, each containing a HindIII restriction enzyme site and two additional nucleotides. A unique HindIII restriction enzyme site in the $r d x A$ fragment in pRDXA-S1 to -S5 and -R1 to $-\mathrm{R} 5$ was used to insert the $\mathrm{Km}$ resistance cassette. To avoid the leaky expression of $r d x A$ due to readthrough from the expression of the $\mathrm{Km}$ resistance cassette, the $\mathrm{Km}$ resistance cassette was subcloned in the reverse direction to the $r d x A$ gene. The direction was confirmed by sequencing the pRDXA series with the $r d x A$ forward primer. The resulting plasmids carrying a $\mathrm{Km}$ resistance cassette were named $\mathrm{pRDXA}(\mathrm{Km})-\mathrm{S} 1$ to $-\mathrm{S} 5$ and $-\mathrm{R} 1$ to $-\mathrm{R} 5$ and used for the $r d x A$ replacement experiments.

\section{Replacement of $r d x A$ by natural transformation}

The G27 H. pylori strain was used for replacement of the endogenous $r d x A$ allele with the $r d x A$ genes from the clinical isolates. Natural transformation with the $\operatorname{pRDXA}(\mathrm{Km})$ series was performed as described $\operatorname{earlier}^{27}$ with the following modification: the G27 strain was struck and incubated for 3 days. Sweeps of G27 colonies from the initial plate were then restruck on a fresh Columbia blood agar plate and incubated. After $24 \mathrm{~h}$, these cells were inoculated as a circle on a fresh Columbia blood agar plate with four 1-inch scrapes. After $6 \mathrm{~h}, 2 \mu \mathrm{g}$ DNA of the pRDXA(Km) series was added to the G27 circle. After $20 \mathrm{~h}$, the whole circle was restreaked onto Columbia blood agar containing $\mathrm{Km}\left(10 \mu \mathrm{g} \mathrm{m}^{-1}\right)$ and incubated for 3-5 days until transformants appeared.

\section{PCR screening for the $r d x A$ replacement of transformants}

Integration of the pRDXA $(\mathrm{Km})$ series in the $r d x A$ locus of G27 H. pylori by single crossover homologous recombination was selected for by $\mathrm{Km}$ resistance (Figure 1). The $r d x A$ replacement of the Km-resistant transformants was confirmed by PCR screening with the $r d x A$ primers as described above and illustrated in Figure 1. If the pRDXA $(\mathrm{Km})$ series were integrated successfully into the wild-type $r d x A$ locus, two bands of $851 \mathrm{bp}(r d x A$ gene expressed by the endogenous $r d x A$ promoter) and $2275 \mathrm{bp}$ (promoterless $r d x A$ gene containing the Km resistance cassette) should be observed. The small PCR band ( $851 \mathrm{bp}$ ) and large band ( $2275 \mathrm{bp}$ ) were extracted and sequenced (Cosmogene Tec.) with the $r d x A$ primers and $\mathrm{Km}$ (seq) primer ( $5^{\prime}$-cgaaagggcctcgtgatacg- $\left.3^{\prime}\right)$ to confirm the single crossover site of homologous recombination, the integration of $r d x A$ of clinically isolated $\mathrm{H}$. pylori and the interruption of G27 $r d x A$ by the $\mathrm{Km}$ resistance cassette (Figure 1).

\section{Reverse transcription-PCR}

To confirm $r d x A$ expression in the R3-R5 clinical $H$. pylori isolates, as well as expression of the integrated $r d x A$ gene and lack of expression of the original G27 endogenous $r d x A$ locus in $H$. pylori transformants, reverse transcription (RT)-PCR was performed on RNA from the R3-R5 clinical $H$. pylori isolates and H. pylori transformants, respectively. Total RNA was isolated using the RNeasy Protect Bacteria Mini kit (Qiagen) according to the manufacturer's instructions. Total RNA was treated with $1 \mathrm{U}$ of RNase-free DNase I (Sigma Chemical Co.) for $15 \mathrm{~min}$, which was then inactivated by adding $50 \mathrm{~mm}$ EDTA 


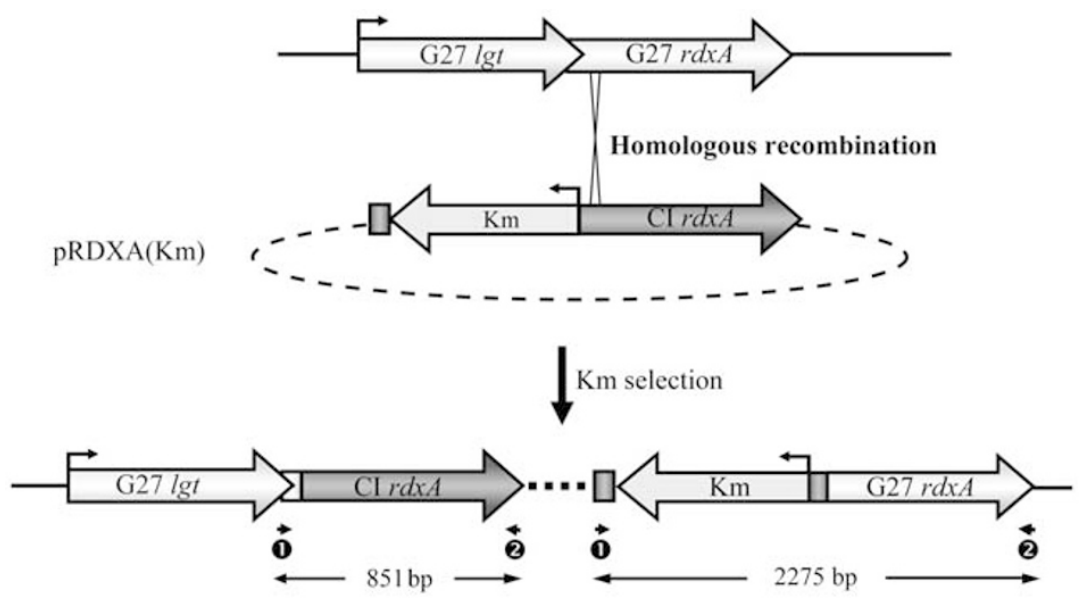

Figure 1 Schematic representation of the integration of $\operatorname{pRDXA}(\mathrm{Km})$ in the $r d x A$ locus of G27 chromosomal DNA by single crossover homologous recombination. In H. pylori, $r d x A$ is transcribed as part of an operon with lgt. ${ }^{25}$ The promoterless $r d x A$ gene from individual clinical isolates (Cls) was amplified by PCR and a kanamycin resistance cassette was inserted into a unique HindlII restriction site within the context of the pRDXA(Km) integration vectors as described in Materials and methods. After transformation of G27 and selection for kanamycin resistance, transformants were screened by PCR to identify those where the pRDXA(Km) plasmid had integrated into the endogenous $r d x A$ locus by homologous recombination. As illustrated in the bottom portion of the figure, two structural $r d x A$ genes exist in the transformants, but only one of the genes can be expressed by the endogenous $r d x A$ promoter and the other one is silent. Promoter elements are indicated by small arrows on the respective open reading frames. The relative position of the primers used to verify proper integration is indicated by the numbers 1 and 2 and the sizes of the indicated PCR products are indicated in base pairs. Items in the figure are not drawn to scale.

Table 1 Deduced RdxA amino-acid changes in MTZ-sensitive and -resistant $\boldsymbol{H}$. pylori isolates

\begin{tabular}{|c|c|c|c|c|c|c|c|c|c|c|c|c|c|c|c|c|c|c|c|}
\hline \multirow{2}{*}{ Strain } & \multirow{2}{*}{$\begin{array}{l}\text { MIC } \\
\left(\mu g \mathrm{ml}^{-1}\right)\end{array}$} & \multicolumn{18}{|c|}{ Amino-acid position and change in $R d x A$} \\
\hline & & 21 & 26 & 37 & 40 & 50 & 52 & 62 & 64 & 88 & 90 & 98 & 106 & 108 & 117 & 131 & 153 & 172 & 206 \\
\hline G27 & 0.75 & $M$ & $Y$ & $A$ & $A$ & $Q$ & $\mathrm{H}$ & $\mathrm{L}$ & $K$ & $S$ & $\mathrm{~K}$ & $G$ & $P$ & $S$ & $A$ & $\mathrm{~K}$ & $\mathrm{~L}$ & V & $A$ \\
\hline S1 & 0.38 & $a^{a}$ & . & . & . & . & . & $\mathrm{V}^{\mathrm{b}}$ & . & $\mathrm{P}$ & $\mathrm{R}$ & S & . & . & . & $\mathrm{R}$ & . & . & . \\
\hline $\mathrm{S} 2$ & 0.25 & . & . & . & . & . & $\mathrm{R}$ & V & . & $P$ & $\mathrm{R}$ & $\mathrm{S}$ & . & $A$ & $S$ & . & . & 1 & . \\
\hline S3 & 0.13 & . & . & . & . & . & $\mathrm{R}$ & . & . & $P$ & $\mathrm{R}$ & S & $S$ & . & $S$ & . & . & . & . \\
\hline S4 & 0.19 & . & . & . & . & . & $\mathrm{R}$ & V & . & $P$ & $\mathrm{R}$ & S & . & . & . & . & . & 1 & . \\
\hline S5 & 0.25 & A & . & . & . & . & $\mathrm{R}$ & V & . & $P$ & $\mathrm{R}$ & $S$ & . & . & . & . & . & 1 & $\mathrm{~T}$ \\
\hline $\mathrm{R} 1$ & 64 & . & . & . & . & $\sim^{c}$ & $\sim$ & $\sim$ & $\sim$ & $\sim$ & $\sim$ & $\sim$ & $\sim$ & $\sim$ & $\sim$ & $\sim$ & $\sim$ & $\sim$ & $\sim$ \\
\hline R2 & 128 & A & . & V & . & . & . & V & . & $\mathrm{P}$ & $\mathrm{R}$ & $\mathrm{S}$ & . & . & . & . & $\sim^{d}$ & $\sim$ & $\sim$ \\
\hline R3 & 256 & . & . & . & . & . & . & V & $\mathrm{N}$ & $P$ & $\mathrm{R}$ & $\mathrm{N}$ & . & . & . & . & . & 1 & . \\
\hline R4 & 256 & . & . & . & . & . & $\mathrm{R}$ & V & . & $P$ & $\mathrm{R}$ & $S$ & . & . & . & . & . & I & $T$ \\
\hline R5 & 256 & . & V & . & $P$ & . & $\mathrm{R}$ & V & . & $P$ & $\mathrm{R}$ & $S$ & . & A & $S$ & . & . & . & . \\
\hline
\end{tabular}

Abbreviations: MIC, minimum inhibitory concentration; MTZ, metronidazole.

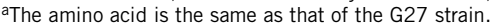

bA missense mutation resulted in the amino-acid substitution.

${ }^{\mathrm{C} A}$ missensense mutation resulted in the amino-acid substitution.

${ }^{d} A$ frame-shift mutation resulted in the stop codon at residue 153 .

and heating to $70^{\circ} \mathrm{C}$ for $10 \mathrm{~min}$. Here, $1 \mu \mathrm{g}$ of total RNA as a template and $10 \mathrm{pm}$ of the $r d x \mathrm{~A}(\mathrm{RT})$ reverse primer $\left(5^{\prime}\right.$-gcattgctctaaaatatagc- $\left.3^{\prime}\right)$ were then used for cDNA synthesis using an RT kit (Bioneer, Daejeon, Republic of Korea) according to the manufacturer's instructions. PCR amplification of the $r d x \mathrm{~A}$ cDNA was then carried out with the $r d x \mathrm{~A}(\mathrm{RT})$ forward primer $\left(5^{\prime}\right.$-ctatcgccaagctcttacaa- $\left.3^{\prime}\right)$ and reverse primer in a DNA thermal cycler (Biometra) using Maxime PCR PreMix (i-StarTaq) (iNtRON Biotechnology, Seongnam-Si, Republic of Korea) as follows: 1 cycle at $95^{\circ} \mathrm{C}$ for $2 \mathrm{~min} ; 35$ cycles at $95^{\circ} \mathrm{C}$ for $30 \mathrm{~s}, 48^{\circ} \mathrm{C}$ for $30 \mathrm{~s}$ and $72{ }^{\circ} \mathrm{C}$ for $30 \mathrm{~s}$ and a final elongation step at $72{ }^{\circ} \mathrm{C}$ for $10 \mathrm{~min}$. The final RT-PCR products ( $297 \mathrm{bp}$ ) were then run on an agarose gel to examine $r d x A$ expression. For the negative control, total RNA of each sample was amplified by PCR without conducting a RT reaction. For these controls, no PCR products were observed, indicating no chromosomal DNA contamination.
To confirm the expression of only the integrated $r d x A$ gene in the $H$. pylori transformants, the final RT-PCR products from the transformants were extracted and sequenced as described above.

\section{RESULTS}

Identification of MTZ-sensitive or -resistant clinical H. pylori isolates

As we wished to elucidate the specific contribution of $r d x A$ and $f r x A$ to MTZ resistance in current clinical $H$. pylori isolates, the MICs of MTZ of several isolates from patients undergoing gastroscopy were first determined by both the E-test and the agar dilution method. For reference, the MTZ MICs were also determined for the well-studied 
Table 2 Deduced FrxA amino-acid changes in MTZ-sensitive and -resistant $\boldsymbol{H}$. pylori isolates

\begin{tabular}{|c|c|c|c|c|c|c|c|c|c|c|c|c|c|c|c|c|c|c|c|c|c|c|c|c|c|c|}
\hline \multirow{2}{*}{ Strain } & \multirow{2}{*}{$\begin{array}{l}M I C \\
\left(\mu g I^{-1}\right)\end{array}$} & \multicolumn{25}{|c|}{ Amino-acid position and change in FrxA } \\
\hline & & 2 & 16 & 18 & 19 & 20 & 21 & 32 & 37 & 43 & 44 & 68 & 72 & 73 & 81 & 103 & 111 & 117 & 124 & 149 & 152 & 153 & 155 & 176 & 193 & 208 \\
\hline G27 & 0.75 & $a^{a}$ & . & $\mathrm{I}^{\mathrm{b}}$ & $\mathrm{R}$ & $S$ & $\sim^{c}$ & $\sim$ & $\sim$ & $\sim$ & $\sim$ & $\sim$ & $\sim$ & $\sim$ & $\sim$ & $\sim$ & $\sim$ & $\sim$ & $\sim$ & $\sim$ & $\sim$ & $\sim$ & $\sim$ & $\sim$ & $\sim$ & $\sim$ \\
\hline S1 & 0.38 & . & $\mathrm{T}$ & . & . & . & . & V & $\mathrm{E}$ & . & $\mathrm{V}$ & . & S & S & 1 & . & . & . & s & . & . & . & $\mathrm{T}$ & . & S & $\mathrm{N}$ \\
\hline S4 & 0.19 & $\mathrm{~V}$ & $\mathrm{~T}$ & . & . & . & . & . & . & $A$ & $\mathrm{~F}$ & . & $\mathrm{S}$ & $\mathrm{S}$ & . & . & $D$ & $M$ & S & K & . & . & . & K & S & . \\
\hline S5 & 0.25 & V & $\mathrm{T}$ & . & . & $\cdot$ & . & . & . & A & $\mathrm{F}$ & . & S & $\mathrm{s}$ & . & . & $\mathrm{D}$ & $M$ & s & . & . & . & . & K & S & . \\
\hline $\mathrm{R} 1$ & 64 & . & $\mathrm{T}$ & . & . & . & . & V & . & $A$ & $\mathrm{~F}$ & . & $S$ & $S$ & . & . & D & $\mathrm{M}$ & S & . & . & . & . & K & S & . \\
\hline R2 & 128 & V & $\mathrm{T}$ & . & . & . & . & . & . & $A$ & $\mathrm{~F}$ & . & $S$ & $S$ & . & . & D & $\mathrm{M}$ & S & . & V & . & . & K & $S$ & . \\
\hline
\end{tabular}

Abbreviations: MIC, minimum inhibitory concentration; MTZ, metronidazole.

${ }^{a}$ The amino acid is the same as that of the 26695 strain.

${ }^{\mathrm{b}} \mathrm{A}$ missense mutation resulted in the amino-acid substitution.

${ }^{\mathrm{C}} \mathrm{A}$ frame-shift mutation resulted in the stop codon at residue 21 .

${ }^{\mathrm{d} A}$ nonsense mutation resulted in the stop codon at residue 68 .

H. pylori strains G27, ATCC43504 and 26695 (Tables 1 and 2). As expected, G27 and 26695 were sensitive to MTZ (MIC, $0.75 \mu \mathrm{g} \mathrm{ml}^{-1}$ ), whereas ATCC43504 was resistant (MIC, $\left.\geqslant 256 \mu \mathrm{g} \mathrm{ml}^{-1}\right)$. Five isolates (S1-S5) that were sensitive to MTZ (MIC, 0.38, 0.25, 0.13, 0.19 and $0.25 \mu \mathrm{g} \mathrm{ml}^{-1}$, respectively) and five isolates (R1-R5) that were resistant to MTZ (MIC, 64, 128 and R3-R5 $\geqslant 256 \mu \mathrm{g} \mathrm{ml}^{-1}$, respectively) were selected for further in-depth analysis (Table 1).

\section{Amino-acid sequences of RdxA and FrxA from MTZ-sensitive and -resistant $H$. pylori isolates}

To identify putative MTZ resistance-associated mutations in $r d x A$ and frx $x$, both genes were sequenced from the $10 \mathrm{H}$. pylori clinical isolates, as well as from the G27 and ATCC43504 reference strains. The DNA sequences obtained for $r d x A$ and $f r x A$ from ATCC43504 were identical to the sequences reported earlier. ${ }^{4,28}$ Moreover, the deduced aminoacid sequence of RdxA and FrxA from the DNA sequence of ATCC43504 indicates that this strain produces truncated non-functional forms of both proteins.

The deduced RdxA amino-acid sequences of G27 and the 10 clinical isolates were compared to identify any potential resistanceassociated nucleotide mutations (Table 1). All of the six MTZ-sensitive strains (G27 and the five clinical isolates) encode full-length RdxA, but each clinical isolate contains 5-8 amino-acid substitutions arising as a result of missense mutations (Table 1). As these strains are all MTZ-sensitive, these amino-acid changes are probably not important for nitroreductase function and hence MTZ resistance. Instead, these variations are likely a by-product of the natural genetic diversity of $H$. pylori, which, in the case of $r d x A$, is estimated to be $5-8 \%{ }^{29,30}$

Two of five MTZ-resistant $H$. pylori isolates (R1 and R2) contained truncated RdxAs due to a nonsense mutation at amino acid 50 and a frameshift resulting in termination at residue 153, respectively. The amino-acid sequence of three (R3-R5) of the MTZ-resistant isolates identified full-length RdxA containing 6-9 amino-acid substitutions. Comparison of the amino-acid sequence between the MTZ-sensitive and -resistant $H$. pylori isolates suggests that substitutions of asparagine at residue 64 and asparagine at residue 98 in the R3 strain, and substitutions of valine at residue 26 and proline at residue 40 in the R5 strain might be important for MTZ resistance. The amino-acid sequence of R4 was virtually identical to that of S5 except for a predicted neutral mutation (Met to Ala) at residue 21 in S5, suggesting that the R4 RdxA is functional similar to the S5 RdxA.

As FrxA has been suggested to play a role in MTZ resistance, we next analyzed the deduced FrxA amino-acid sequences to identify any amino-acid changes. As FrxA is truncated in G27, the FrxA aminoacid sequence from 26695 was used to compare the FrxA sequence of the 10 clinical isolates (Table 2). All five of the MTZ-sensitive H. pylori strains encoded full-length FrxA containing 5-12 amino-acid substitutions by missense mutations. Among the five MTZ-resistant $H$. pylori isolates, R4 encodes a truncated FrxA due to a nonsense mutation at residue 68 . The amino-acid sequences of the four remaining MTZ-resistant isolates (R1, R2, R3 and R5) showed fulllength FrxA containing 10-12 amino-acid substitutions by missense mutations. Surprisingly, no putative MTZ resistance-associated missense mutations of fr $x A$ were identified as no unique missense mutation in the MTZ-resistant strains was identified, as compared with the MTZ-sensitive strains. The one exception was a neutral mutation (Ala to Val) at residue 152 or 153. It is noted that the FrxA sequence from G27 showed that this MTZ-sensitive strain, which encodes a full-length RdxA protein, contained a truncated FrxA due to a frameshift mutation at residue 21 . This strongly suggests that truncation of frxA alone does not result in MTZ resistance. Given that mutation of frxA was not sufficient to impart MTZ resistance, we focused the remainder of our studies on the role of $r d x A$ mutations on MTZ resistance.

Analysis of clinical $r d x A$ alleles in an isogenic $H$. pylori background To directly evaluate the contribution of the truncated $r d x A$ and substituted $r d x A$ alleles to MTZ resistance in an isogenic strain background, the $r d x A$ locus of MTZ-sensitive wild-type $H$. pylori was genetically replaced with the $r d x A$ gene of several of the clinical isolates (Figure 1). G27 was chosen as the recipient for the $r d x A$ replacement because G27 is MTZ sensitive (MIC, $0.75 \mu \mathrm{g} \mathrm{ml}^{-1}$ ), is capable of natural transformation and encodes a truncated FrxA 


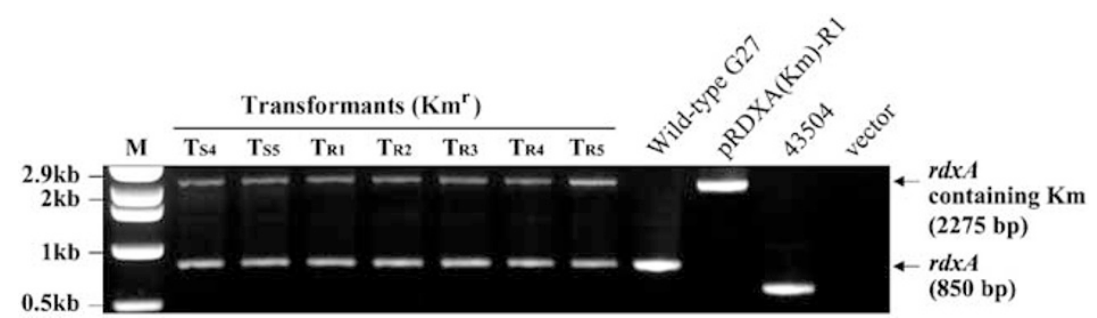

Figure 2 PCR screening for the $r d x A$ replacement of transformants. The $r d x A$ replacement of $\mathrm{Km}$-resistant $H$. pylori transformant was confirmed by PCR screening with an $r d x A$ primer set. Transformants showed two bands-an $851 \mathrm{bp}$ ( $r d x A$ gene) and a $2275 \mathrm{bp}$ ( $r d x A$ containing the Km resistance cassette).

protein. We considered the truncation of FrxA to be important as an earlier study suggested that combined inactivation of FrxA and RdxA enhances MTZ resistance. ${ }^{21}$ The $r d x A$ gene from S4, S5 and R1-R5 were introduced into $\mathrm{G} 27$ by natural transformation with pRDXA(km)-S4, -S5 and -R1-R5 (Figure 1). The S4 and S5 $r d x A$ alleles were selected as representative forms of $r d x A$ from the MTZsensitive strains. The $\mathrm{pRDXA}(\mathrm{km})$ plasmid series does not replicate in H. pylori, and thus to obtain the $\mathrm{Km}$ resistance phenotype, the plasmid must integrate into the $r d x A$ chromosomal locus by single crossover homologous recombination (Figure 1). Thus, after transformation a merodiploid is produced where only the introduced $r d x A$ is expressed by the endogenous $r d x A$ promoter. To avoid the leaky expression of $r d x A$ from the $\mathrm{Km}$ resistance cassette promoter, the $\mathrm{Km}$ resistance cassette was subcloned in the reverse orientation to the $r d x A$ gene (Figure 1).

The proper $r d x A$ replacement in the Km-resistant transformants was confirmed by PCR with the $r d x A$ primer set that showed the following two bands; an 851-bp band ( $r d x A$ gene expressed from the endogenous promoter) and a 2275-bp band (a promoterless $r d x A$ containing the $\mathrm{Km}$ resistance cassette) (Figures 1 and 2). In addition to the PCR analysis, the expressing $r d x A$ gene (small PCR product) was sequenced to confirm the single crossover site and the complete replacement with the clinical isolates $r d x A$ locus (data not shown). Finally, interruption of the native G27 $r d x$ A gene by the Km resistance cassette was confirmed by sequencing of the large PCR product (2275 bp) (data not shown). For all natural transformations, we isolated transformants in which the single crossover occurred upstream from the first identified amino-acid change. Therefore, the clinical isolates $r d x A$ allele completely replaced the G27 $r d x A$ gene. At least three individual transformants from each complete replacement were obtained and analyzed further.

\section{Verification of expression of the introduced $r d x \mathrm{~A}$ gene but not the G27 endogenous $r d x \mathrm{~A}$}

To confirm that the $r d x A$ expressed by each of our merodiploid strains was indeed from the integrated copy of $r d x A$ from our clinical isolates, we performed RT-PCR for the $r d x A$ locus using total RNA harvested from the $H$. pylori transformants. On the basis of the assumption that the sequence of the cDNA produced from these RT reactions would show whether the integrated $r d x A$ or endogenous $r d x A$ was being expressed, we compared these cDNA sequences to the sequences we obtained earlier (Table 1). Total RNA from each sample was also amplified by PCR without first conducting a RT reaction and no PCR products were observed, indicating that there was no chromosomal DNA contamination (data not shown).

The RT-PCR products of R1-R5 were each extracted from an agarose gel and sequenced. The sequence analysis indicated that each $H$. pylori transformant indeed expressed only the integrated $r d x A$ from the

\section{Table 3 MTZ MICs of transformants}

\begin{tabular}{ll}
\hline Clinical isolates (MICs) & Transformants (MICs) \\
\hline S4 $\left(0.19 \mu \mathrm{g} \mathrm{ml}^{-1}\right)$ & $\mathrm{T}_{\mathrm{S} 4}\left(0.25-0.75 \mu \mathrm{g} \mathrm{ml}^{-1}\right)$ \\
S5 $\left(0.25 \mu \mathrm{gl}^{-1}\right)$ & $\mathrm{T}_{\mathrm{S} 5}\left(0.25-0.35 \mu \mathrm{gl}^{-1}\right)$ \\
$\mathrm{R} 1\left(64 \mu \mathrm{gl}^{-1}\right)$ & $\mathrm{T}_{\mathrm{R} 1}\left(\geqslant 256 \mu \mathrm{gl}^{-1}\right)$ \\
R2 $\left(128 \mu \mathrm{g} \mathrm{m}^{-1}\right)$ & $\mathrm{T}_{\mathrm{R} 2}\left(\geqslant 256 \mu \mathrm{gl}^{-1}\right)$ \\
$\mathrm{R} 3\left(\geqslant 256 \mu \mathrm{gl}^{-1}\right)$ & $\mathrm{T}_{\mathrm{R} 3}\left(0.25-0.75 \mu \mathrm{gl}^{-1}\right)$ \\
$\mathrm{R} 4\left(\geqslant 256 \mu \mathrm{gl}^{-1}\right)$ & $\mathrm{T}_{\mathrm{R} 4}\left(0.25-3 \mu \mathrm{ml}^{-1}\right)$ \\
R5 $\left(\geqslant 256 \mu \mathrm{gl}^{-1}\right)$ & $\mathrm{T}_{\mathrm{R} 5}\left(0.25-3 \mu \mathrm{gl}^{-1}\right)$
\end{tabular}

Abbreviations: MIC, minimum inhibitory concentration; MTZ, metronidazole. The $r d x$ A replacement of MTZ-sensitive G27 (MIC, $0.75 \mu \mathrm{g} \mathrm{ml}^{-1}$ ) with the $r d x A s$ of the clinical isolates resulted in the following MTZ MICs.

clinical $H$. pylori isolates as the expression of the disrupted original G27 $r d x$ A could not be detected.

\section{MIC determination of $r d x A$-replaced $H$. pylori transformants}

To determine whether the MTZ resistance/sensitivity profile of the clinical isolates was transferred by the expression of the clinical $r d x A$ locus in the isogenic strain background, the MTZ MICs of the $r d x A$ replaced $H$. pylori transformants were determined by both the E-test and agar dilution method. As shown in Table 3, replacement with the $r d x A$ genes from the MTZ-sensitive strains (S4 and S5) resulted in $H$. pylori transformants that remained sensitive to MTZ (MIC, 0.75 and $0.35 \mu \mathrm{g} \mathrm{ml}^{-1}$, respectively). This was expected as the RdxAs of the MTZ-sensitive strains should be functional. The MICs were similar to that of the recipient strain, G27 (MIC, $0.75 \mu \mathrm{g} \mathrm{ml}^{-1}$ ). Replacement with the $r d x A$ genes from the MTZ-resistant strains (R1-R5) resulted in a wide range of MICs $\left(0.25-\geqslant 256 \mu \mathrm{g} \mathrm{ml}^{-1}\right)$. As expected, replacement with $r d x A$ from $\mathrm{R} 1$ and R2 resulted in high resistance to MTZ (MIC, $\geqslant 256 \mu \mathrm{g} \mathrm{ml}^{-1}$ ). These results indicate that truncated, and hence non-functional, RdxA causes MTZ resistance in $H$. pylori. In addition, the MICs were higher than those of the original clinical isolated $H$. pylori strains (R1 MIC, $64 \mu \mathrm{g} \mathrm{ml}^{-1}$ and $\mathrm{R} 2 \mathrm{MIC}$, $\left.128 \mu \mathrm{g} \mathrm{ml}^{-1}\right)$. This is in keeping with the idea that the truncated FrxA already found in G27 can enhance MTZ resistance in the presence of a non-functional $r d x A$ mutation. Unexpectedly, replacement with the $r d x A$ genes from the MTZ-resistant strains R3, R4 and R5 still resulted in sensitivity to MTZ (MIC, $0.25-0.75,0.25-3$ and $0.25-3 \mu \mathrm{g} \mathrm{ml}^{-1}$, respectively). We originally predicted that the asparagine substitution at residue 64 and asparagine at residue 98 in the R3 strain, and the valine at residue 26 and proline at residue 40 in the R5 might be important for MTZ resistance (Table 1). However, the fact that the $r d x A$ replacement containing these amino acids still resulted 


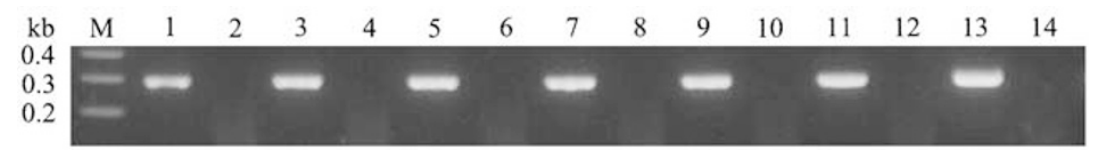

Figure 3 Reverse transcription (RT)-PCR to confirm $r d x A$ expression in metronidazole (MTZ)-sensitive and -resistant $H$. pylori strains. Total RNA was isolated from the indicated strains, treated with DNase and then used to examine $r d x A$ expression by RT-PCR as detailed in Materials and methods. Each of the strains expressed $r d x A$ as they yielded the expected 297-bp product. For the negative control, total RNA from each sample was amplified by PCR without first conducting a reverse transcription reaction. No PCR products were observed, indicating no chromosomal DNA contamination. Lanes M=Marker, $1=\mathrm{G} 27$, 2=G27 no RT, 3=S3, 4=S3 no RT, 5=S4, 6=S4 no RT, 7=S5, 8=S5 no RT, 9=R3, 10=R3 no RT, 11=R4, 12=R4 no RT, 13=R5 and 14=R5 no RT.

Table 4 Sequence variation in the upstream region of $r d x A$ in $H$. pylori clinical isolates

\begin{tabular}{|c|c|c|}
\hline \multirow{2}{*}{ Strain } & \multicolumn{2}{|l|}{ Sequence of rdxA upstream region } \\
\hline & $S D^{\mathrm{a}}$ sequence & $\begin{array}{l}\text { Start } \\
\text { codon }\end{array}$ \\
\hline \multicolumn{3}{|c|}{ HP2600G CTA C G A A A AT TC TA A A A A ATA A A G G A A A ATC A $\boldsymbol{A} \boldsymbol{T} \boldsymbol{G}$} \\
\hline G27 & GCTACAAGGCATTCTAAAAAAATA A A G G A A & A ATG \\
\hline S1 & GCTACGAAAAATTCTAAAAAAATA A A G G G A A & $A \boldsymbol{A T G}$ \\
\hline S2 & GCTACGAAAAATTCTAAAAAAATA A AG GAAA & A $A T G$ \\
\hline S3 & GCTACGAAAAATTCTAAAAAAATAAAG GAAA & A $A T G$ \\
\hline S4 & GCTACGAAAAATTCTAAAAAAATA A AG GAAA & A $A T G$ \\
\hline S5 & GCTACGAAAAATTCTAAAAAAATA A AGG AAA & A $\boldsymbol{A T G}$ \\
\hline R1 & GCTAC- ${ }^{b} A A A A A T T C T A A A A A A A T A \mathbf{A A G G A A A}$ & A ATG \\
\hline R2 & GCTACGAAAAATTCTAAAAAAATA A A G G G A A & A $\boldsymbol{A T G}$ \\
\hline R3 & GCTACGAAAAATTCTAAAAAAATA A AGGAAA & A $A T G$ \\
\hline R4 & GCTACGAAAAATTCTAAAAAAATA A AGGAAA & A $A T G$ \\
\hline R5 & GCTAC-AAAAATTCTAAAAAAATAAAGGAAA & A $A T G$ \\
\hline
\end{tabular}

Gray blocks indicate differences from the sequence of HP2600

RdxA start codon (ATG) is indicated in italic bold

aSD (Shine-Dalgarno) sequence (AAGGAA) is indicated in bold.

$b_{-}$indicates a nucleotide deletion.

in MTZ sensitivity suggests that these substitutions are not significant for MTZ resistance and thus reductase function.

\section{Verification of $r d x A$ expression in the clinical isolates}

As we examined expression of the clinical $r d x A$ allele in G27 using the G27 promoter to drive expression of the transferred $r d x A$ gene, we sought to ensure that the R3-R5 clinical isolates did not contain any promoter mutations that prevented $r d x A$ expression in the context of the clinical strain background. Therefore, $r d x A$ expression was examined by RT-PCR in the R3-R5 clinical isolates as well as in G27. This analysis showed that each of the strains expressed $r d x A$ (Figure 3 ). Therefore, any variations in the upstream sequences in R3-R5 do not prevent $r d x A$ expression and thus, do not explain the obtained MTZ resistance profiles of the clinical isolates.

Finally, as R3-R5 apparently encode functional RdxA and as mutations upstream of the RdxA start codon have been identified that affect MTZ resistance, ${ }^{21}$ we investigated whether there were any nucleotide mutations in this region that might affect RdxA translation in the original R3-R5 clinical isolates. To examine sequence polymorphisms, the region upstream of the ATG start codon, which includes the Shine-Dalgarno sequence of $r d x A$, were sequenced in all 10 clinical isolates (Table 4). The sequences were then compared with that of HP2600 28 and G27, which are both sensitive to MTZ. As all the clinical isolates showed sequences that were most similar to HP2600, we focused our comparison to this MTZ-sensitive strain. Because the sequences of S2-S5 and R3 and R4 were the same as that of HP2600 there are no changes that can account for the MTZ resistance seen in R3 and R4. In addition, the sequences of S1 and $\mathrm{R} 2$ were identical, suggesting that the single nucleotide difference from the HP2600 sequence is not important for MTZ resistance. The sequence of R1 and R5 showed a one nucleotide $(G)$ deletion, which might affect $r d x A$ expression; however, the sequence of G27 has four nucleotide variations in this same region. As G27 is sensitive to MTZ, it seems unlikely that these variations should affect $r d x A$ expression in R1 and R5.

\section{DISCUSSION}

This study examined the resistance of $H$. pylori to the anti-microbial agent MTZ and specifically focused on the contribution of the $r d x A$ and frxA genes to MTZ resistance. Five MTZ-sensitive and five MTZresistant $H$. pylori strains were obtained from the clinical isolates. Analysis of the deduced RdxA and FrxA amino-acid sequences of these isolates revealed various mutations. To directly evaluate the contribution of these specific $r d x A$ mutations to MTZ resistance, we introduced these $r d x A$ variants into G27 while inactivating its own $r d x A$ gene. Our results show that (i) premature truncation of frxA alone is not sufficient to cause MTZ resistance in $H$. pylori, but has the capacity to enhance MTZ resistance in $H$. pylori containing a deficient $r d x A$; (ii) introduction of an inactivated $r d x A$ (non-functional premature truncation) is sufficient to confer MTZ resistance to G27 (normally MTZ sensitive); (iii) introduction of the missense-mutated $r d x A$ alleles (6-9 amino-acid substitutions each) does not result in MTZ resistance; (iv) three high MTZ-resistant isolates (MIC, $\geqslant 256 \mu \mathrm{g} \mathrm{ml}^{-1}$ ) have functional $r d x A$, suggesting that an MTZ resistance phenotype can arise in $H$. pylori in the absence of inactivating mutations in $r d x A$.

MTZ resistance associated with mutation of $f r x A$ is still one of the most controversial topics. Kwon et al..$^{22}$ reported that $f r x A$ inactivation resulted in MICs ( 32 or $128 \mu \mathrm{g} \mathrm{ml}^{-1}$ ) similar to those seen with $r d x A$ inactivation, whereas a study by Jeong et al. ${ }^{21}$ showed that frxA inactivation enhanced MTZ resistance in $r d x A$-deficient $H$. pylori but had little effect on the MTZ susceptibility of strains carrying a functional $r d x A$ allele. In addition, Yang et al. ${ }^{23}$ recently identified the truncation of FrxA in an MTZ-sensitive H. pylori isolate. Our results agree with the studies of the Jeong and Yang groups. MTZ-sensitive G27 (MIC, $0.75 \mu \mathrm{g} \mathrm{ml}^{-1}$ ) contains a truncated FrxA due to a frameshift-causing termination at residue 21 . As full-length FrxA is 217 amino acids, the truncated FrxA is most likely non-functional. Therefore, the functional inactivation of FrxA alone cannot induce MTZ resistance in this strain. In addition, the comparison of FrxA aminoacid sequences (Table 2) shows that none of the putative missense mutations is likely to be responsible for MTZ resistance as no unique missense mutations in the MTZ-resistant strains were identified with the exception of a neutral mutation (Ala to Val) at residue 152 or 153. It is worth noting that the replacement of G27 $r d x A$ with the nonsense-mutated $r d x A$ (premature truncation) from R1 (MIC, $64 \mu \mathrm{g} \mathrm{ml}^{-1}$ ) and the frameshift-mutated $r d x A$ (premature truncation) 
from R2 (MIC, $128 \mu \mathrm{g} \mathrm{ml}^{-1}$ ) resulted in higher MTZ resistance (MIC, $\geqslant 256 \mu \mathrm{g} \mathrm{ml}^{-1}$ ) than in the original isolates. Although this comparison is between $H$. pylori strains of different genetic backgrounds, this result is probably due to double functional inactivation of RdxA and FrxA in the transformants as G27 contains a non-functional FrxA and both R1 and R2 isolates contain full-length and presumably functional FrxA (Table 2). This fact suggests that FrxA inactivation does indeed enhance MTZ resistance in $r d x A$-deficient $H$. pylori and is in agreement with the study of Jeong et al. ${ }^{21}$

Several $r d x A$ replacements were performed to evaluate the role of $r d x A$ mutations in MTZ resistance. We designed our study such that the replaced $r d x A$ s of all transformants were expressed by the same G27 endogenous $r d x A$ promoter. This design enabled us to avoid the potential problem of differential promoter regulation and as a result, all transformants are in the same genetic background. Earlier, presumably important mutations of $r d x A$ for MTZ resistance were simply identified by comparison of the RdxA sequence between MTZsensitive and -resistant $H$. pylori strains. ${ }^{2,31,32}$ However, these mutations were not evaluated directly for their contribution to MTZ resistance. Comparative analysis of the $\mathrm{RdxA}$ sequences in our study predicted that the changes of Lys $64 \rightarrow$ Asn and Gly98 $\rightarrow$ Asn in the R3 strain, and Tyr26 $\rightarrow$ Val and Ala $40 \rightarrow$ Pro in the R5 strain might be responsible for MTZ resistance. $r d x A$ replacement proved that these substitutions are not critical changes in MTZ resistance as the G27 strain carrying them remained MTZ sensitive. These findings suggest that the amino-acid changes should be attributed to natural genetic diversity and are not associated with MTZ resistance. Recent studies revealed the RdxA amino-acid changes of Arg10 $\rightarrow$ Lys, Arg16 $\rightarrow$ His, Met2 $1 \rightarrow$ Ala, His53 $\rightarrow$ Arg, Met56 $\rightarrow$ Ile, Leu62 $\rightarrow$ Val, Ala68 $\rightarrow$ Val, Gly98 $\rightarrow$ Ser, Gly163 $\rightarrow$ Asp and Ala206 $\rightarrow$ Thr in MTZ-resistant strains, ${ }^{2,31,32}$ suggesting that those substitutions may be important in MTZ resistance. However, in our study the replacement with the missense-mutated $\quad r d x A$ containing amino-acid substitutions $($ Met21 $\rightarrow$ Ala, Leu62 $\rightarrow$ Val, Gly98 $\rightarrow$ Ser and Ala206 $\rightarrow$ Thr) failed to transfer the MTZ-resistant phenotype, suggesting that the substitutions are not important for MTZ resistance. In addition, only Paul et al. ${ }^{32}$ confirmed experimentally that RdxA amino-acid substitutions (Cys19 $\rightarrow$ Tyr and Thr49 $\rightarrow$ Lys) were causative for MTZ resistance. In that study, MTZ-sensitive strains were transformed with PCR products of MTZ-resistant $r d x A$ and the appearance of MTZ-resistant isolates was selected for studies on MTZ. MTZ-resistant transformants were then analyzed for their $r d x A$ sequences. It should be noted that it is formally possible that the selective pressure of MTZ applied in that study forced other mutations to arise elsewhere that result in MTZ resistance. The PCR-transformed strains were not screened for putative resistance-associated mutations in other genes. To prevent MTZselective pressure in our study, the transformants were selected by $\mathrm{Km}$ resistance and MTZ MICs were then measured. In the future, it will be interesting to directly assess the role of the RdxA substitutions identified by Paul et al. ${ }^{32}$ for their significance in MTZ resistance using the $r d x A$ replacement strategy.

Three clinical isolates (R3-R5) showed high resistance to MTZ (MIC, $\geqslant 256 \mu \mathrm{g} \mathrm{ml}^{-1}$ ), even though our data indicate that they have functional $r d x A$. This result is in agreement with Marais et al. ${ }^{33}$ who suggested that an MTZ resistance phenotype may arise in $H$. pylori without mutation in $r d x A$ or $f r x A$. Interestingly, several groups $s^{22,34-37}$ and genome sequence annotation ${ }^{38-40}$ suggest other putative redox systems that may play a role in MTZ resistance. These include $f d x B$ (encoding a ferredoxin-like protein), $f d x A$ (ferredoxin), fldA (flavodoxin), oorD (the $\gamma$-subunit of 2-oxoglutarate oxidoreductase and por $D$ (the $\gamma$-subunit of pyruvate ferredoxin oxidoreductase). However, with the exception of $f d x B$, research to identify other MTZ important redox systems has been hampered because the deletion of the genes seems to be lethal for $H$. pylori. $^{22}$ Recently, van Amsterdam et al. ${ }^{41}$ reported that double mutation of HP0605 and HP0971 (TolC-like proteins) results in decreased MTZ resistance (from wild-type MIC, $>256 \mu \mathrm{g} \mathrm{ml}^{-1}$ to $8 \mu \mathrm{g} \mathrm{ml}^{-1}$ ), indicating that the TolC efflux pump may confer resistance to MTZ. Therefore, $H$. pylori possessing TolC efflux pumps may be resistant to MTZ independent of $r d x A$ or frxA mutations.

In summary, the findings of this study clarify some of the debate surrounding the questions of whether deletion of frxA alone can induce MTZ resistance or simply enhance the resistance in an $r d x A$ deficient $H$. pylori strain. Additionally, our $r d x A$ replacement approach provides some of the first analysis of the role of specific mutations in MTZ resistance. Our results show that deletion of $r d x A$ induces MTZ resistance, but that none of the 15 amino-acid substitutions found in $r d x A$ of the MTZ-resistant strains is able to cause MTZ resistance. This strongly suggests that the substitution mutations identified previously by sequence comparison need to be directly analyzed for their contribution to MTZ resistance. Finally, MTZ resistance in $H$. pylori can arise without mutations in $r d x A$ or $f r x A$, clearly suggesting that other genetic elements are involved in MTZ resistance. Future work from our group will focus on the identification of the genes responsible for this MTZ resistance phenotype.

\section{ACKNOWLEDGEMENTS}

We thank Joong-Chul Lee for the gift of plasmid pILL600, Doug Berg for helpful suggestions and H Gancz and B Carpenter for a critical reading of the paper. This study was supported by the Korea Research Foundation Grant, which was funded by the Korean Government (MOEHRD) (KRF-2006-311-E00083).

1 Warren, J. R. \& Marshall, B. J. Unidentified curved bacilli on gastric epithelium in active chronic gastritis. Lancet 1, 1273-1275 (1983).

2 Bereswill, S., Krainick, C., Stahler, F., Herrmann, L. \& Kist, M. Analysis of the rdxA gene in high-level metronidazole-resistant clinical isolates confirms a limited use of $r d x A$ mutation as a marker for prediction of metronodazole resistance in Helicobacter pylori. FEMS Immunol. Med. Microbiol. 36, 193-198 (2003)

3 Crump, M., Gospodarowicz, M. \& Shepherd, F. A. Lymphoma of the gastrointestinal tract. Semin. Oncol. 26, 324-337 (1999).

4 Debets-Ossenkopp, Y. J. et al. Insertion of mini-IS605 and deletion of adjacent sequence in the nitroreductase $(\mathrm{rdxA})$ gene cause metronidazole resistance in Helicobacter pylori NCTC11637. Antimicrob. Agents Chemother. 43, 2657-2663 (1999).

5 Parsonnet, J. Helicobacter pylori. Infect. Dis. Clin. North Am. 12, 185-197 (1998)

6 Parsonnet, J. Helicobacter pylori: the size of the problem. Gut 43, S6-S9 (1998).

7 Chiba, N., Rao, B. V., Rademaker, J. W. \& Hunt, R. H. Meta-analysis of the efficacy of antibiotic therapy in eradicating Helicobacter pylori. Am. J. Gastroenterol. 87, 1716-1727 (1992).

8 Houben, M. H. et al. A systematic review of Helicobacter pylori eradication therapythe impact of antimicrobial resistance on eradication rates. Aliment. Pharmacol. Ther. 13, 1047-1055 (1999).

9 Glupczynski, Y. Antimicrobial resistance in Helicobacter pylori: a global overview. Acta. Gastroenterol. Belg. 61, 357-366 (1998).

10 Miyaji, H. et al. Susceptibility of Helicobacter pylori isolates to metronidazole, clarithromycin and amoxycillin in vitro and in clinical treatment in Japan. Aliment. Pharmacol. Ther. 11, 1131-1136 (1997).

11 Goddard, A. F. \& Logan, R. P. Antimicrobial resistance and Helicobacter pylori. J. Antimicrob. Chemother. 37, 639-643 (1996).

12 Mégraud, F. et al. Antimicrobial susceptibility testing of Helicobacter pylori in a large multicenter trial: the MACH 2 study. Antimicrob. Agents Chemother. 43, 2747-2752 (1999).

13 Edwards, D. I. Nitroimidazole drugs - action and resistance mechanisms. I. Mechanisms of action. J. Antimicrob. Chemother. 31, 9-20 (1993).

14 Samuelson, J. Why metronidazole is active against both bacteria and parasites. Antimicrob. Agents Chemother. 43, 1533-1541 (1999).

15 Townson, S. M., Boreham, P. F., Upcroft, P. \& Upcroft, J. A. Resistance to the nitroheterocyclic drugs. Acta. Trop. 56, 173-194 (1994).

16 Edwards, D. I. Nitroimidazole drugs-action and resistance mechanisms. II. Mechanisms of resistance. J. Antimicrob. Chemother. 31, 201-210 (1993). 
17 Lockerby, D. L., Rabin, H. R. \& Laishley, E. J. Role of the phosphoroclastic reaction of Clostridium pasteurianum in the reduction of metronidazole. Antimicrob. Agents Chemother. 27, 863-867 (1985).

18 Declerck, P. J., De Ranter, C. J. \& Volckaert, G. Base specific interaction of reductively activated nitroimidazoles with DNA. FEBS Lett. 164, 145-148 (1983).

19 Declerck, P. J. \& De Ranter, C. J. In vitro reductive activation of nitroimidazoles. Biochem. Pharmacol. 35, 59-61 (1986).

20 Kedderis, G. L., Argenbright, L. S. \& Miwa, G. T. Mechanism of reductive activation of a 5-nitroimidazole by flavoproteins: model studies with dithionite. Arch. Biochem. Biophys. 262, 40-48 (1988).

21 Jeong, J. Y. et al. Sequential inactivation of rdxA (HP0954) and frxA (HP0642) nitroreductase genes causes moderate and high-level metronidazole resistance in Helicobacter pylori. J. Bacteriol. 182, 5082-5090 (2000).

22 Kwon, D. H. et al. Analysis of $\mathrm{rdxA}$ and involvement of additional genes encoding $\mathrm{NAD}(\mathrm{P}) \mathrm{H}$ flavin oxidoreductase ( $\mathrm{FrXA}$ ) and ferredoxin-like protein $(\mathrm{FdxB})$ in metronidazole resistance of Helicobacter pylori. Antimicrob. Agents Chemother. 44, 2133-2142 (2000).

23 Yang, Y. J., Wu, J. J., Sheu, B. S., Kao, A. W. \& Huang, A. H. The rdxA gene plays a more major role than frxA gene mutation in high-level metronidazole resistance of Helicobacter pylori in Taiwan. Helicobacter. 9, 400-407 (2004).

24 Xiang, Z. et al. Analysis of expression of CagA and VacA virulence factors in 43 strains of Helicobacter pylori reveals that clinical isolates can be divided into two major types and that CagA is not necessary for expression of the vacuolating cytotoxin. Infect. Immun. 63, 94-98 (1995).

25 Jeong, J. Y., Mukhopadhyay, A. K., Akada, J. K., Dailidiene, D., Hoffman, P. S. \& Berg, D. E. Roles of FrxA and RdxA nitroreductases of Helicobacter pylori in susceptibility and resistance to metronidazole. J. Bacteriol. 183, 5155-5162 (2001).

26 Ferrero, R. L., Cussac, V., Courcoux, P. \& Labigne, A. Construction of isogenic ureasenegative mutants of Helicobacter pylori by allelic exchange. J. Bacteriol. 174, 4212-4217 (1992).

27 Clayton, C. L. \& Mobley, H. L. T. Helicobacter pylori Protocols (Humana Press, Totowa, 1997).

28 Kwon, D. H., Kato, M., El-Zaatari, F. A., Osato, M. S. \& Graham, D. Y. Frame-shift mutations in $\mathrm{NAD}(\mathrm{P}) \mathrm{H}$ flavin oxidoreductase encoding gene (frxA) from metronidazole resistant Helicobacter pylori ATCC43504 and its involvement in metronidazole resistance. FEMS Microbiol. Lett. 188, 197-202 (2000).
29 Mendz, G. L. \& Megraud, F. Is the molecular basis of metronidazole resistance in microaerophilic organisms understood? Trends Microbiol. 10, 370-375 (2002).

30 Solca, N. M., Bernasconi, M. V. \& Piffaretti, J. C. Mechanism of metronidazole resistance in Helicobacter pylori: comparison of the rdxA gene sequences in 30 strains. Antimicrob. Agents Chemother. 44, 2207-2210 (2000).

$31 \mathrm{Kwon}, \mathrm{D}$. H. et al. DNA sequence analysis of $\mathrm{rdxA}$ and frxA from 12 pairs of metronidazole-sensitive and -resistant clinical Helicobacter pylori isolates. Antimicrob. Agents Chemother. 45, 2609-2615 (2001).

32 Paul, R., Postius, S., Melchers, K. \& Schafer, K. P. Mutation of the Helicobacter pylori genes $\mathrm{rdxA}$ and pbp1 cause resistance against metronidazole and amoxicillin. Antimicrob. Agents Chemother. 45, 962-965 (2001).

33 Marais, A., Bilardi, C., Cantet, F., Mendz, G. L. \& Megraud, F. Characterization of the genes rdxA and frxA involved in metronidazole resistance in Helicobacter pylori. Res. Microbiol. 154, 137-144 (2003).

34 Hoffman, P. S., Goodwin, A., Johnsen, J., Magee, K. \& Veldhuyzen van Zanten, S. J. Metabolic activities of metronidazole-sensitive and -resistant strains of Helicobacter pylori: repression of pyruvate oxidoreductase and expression of isocitrate lyase activity correlate with resistance. J. Bacteriol. 178, 4822-4829 (1996).

35 Jorgensen, M. A., Trend, M. A., Hazell, S. L. \& Mendz, G. L. Potential involvement of several nitroreductases in metronidazole resistance in Helicobacter pylori. Arch. Biochem. Biophys. 392, 180-191 (2001).

36 Kaihovaara, P., Hook-Nikanne, J., Uusi-Oukari, M., Kosunen, T. U. \& Salaspuro, M. Flavodoxin-dependent pyruvate oxidation, acetate production and metronidazole reduction by Helicobacter pylori. J. Antimicrob. Chemother. 41, 171-177 (1998).

37 Trend, M. A., Jorgensen, M. A., Hazell, S. L. \& Mendz, G. L. Oxidases and reductases are involved in metronidazole sensitivity in Helicobacter pylori. Int. J. Biochem. Cell. Biol. 33, 143-153 (2001).

$38 \mathrm{Alm}, \mathrm{R}$. A. et al. Genomic-sequence comparison of two unrelated isolates of the human gastric pathogen Helicobacter pylori. Nature 397, 176-180 (1999).

39 Marais, A., Mendz, G. L., Hazell, S. L. \& Megraud, F. Metabolism and genetics of Helicobacter pylori: the genome era. Microbiol. Mol. Biol. Rev. 63, 642-674 (1999).

$40 \mathrm{Tomb}$, J. F. et al. The complete genome sequence of the gastric pathogen Helicobacter pylori. Nature 388, 539-547 (1997).

41 van Amsterdam, K., Bart, A. \& van der Ende, A. A Helicobacter pylori TolC efflux pump confers resistance to metronidazole. Antimicrob. Agents Chemother. 49, 1477-1482 (2005). 